\title{
Electricity Network Reliability Planning and Evaluation Considering Flexible Solutions
}

Link to publication record in Manchester Research Explorer

\section{Citation for published version (APA):}

Tumelo-Chakonta, C., Kapetanaki, A., \& Kopsidas, K. (2014). Electricity Network Reliability Planning and Evaluation Considering Flexible Solutions. In 9th Mediterranean Conference on Power Generation, Transmission, Distribution and Energy Conversion (MedPower) Institution of Engineering and Technology .

\section{Published in:}

9th Mediterranean Conference on Power Generation, Transmission, Distribution and Energy Conversion (MedPower)

\section{Citing this paper}

Please note that where the full-text provided on Manchester Research Explorer is the Author Accepted Manuscript or Proof version this may differ from the final Published version. If citing, it is advised that you check and use the publisher's definitive version.

\section{General rights}

Copyright and moral rights for the publications made accessible in the Research Explorer are retained by the authors and/or other copyright owners and it is a condition of accessing publications that users recognise and abide by the legal requirements associated with these rights.

\section{Takedown policy}

If you believe that this document breaches copyright please refer to the University of Manchester's Takedown Procedures [http://man.ac.uk/04Y6Bo] or contact uml.scholarlycommunications@manchester.ac.uk providing relevant details, so we can investigate your claim.

\section{OPEN ACCESS}




\title{
Electricity Network Reliability Planning and Evaluation Considering Flexible Solutions
}

\author{
C.Tumelo-Chakonta, Student Member IEEE, A. Kapetanaki, Student Member IEEE, Konstantinos Kopsidas, Member IEEE
}

Abstract-Amid contemporary electric power system planning and operating regimes, the transmission network is pervasively operated, and planned, with time-invariant thermal ratings (TITRs) of transmission assets. This results in a transmission network which is not fully (and efficiently) utilized. In the future, however, power systems will be increasingly expected to transition into a smart grid-in order to be more efficient and economical. Consequently, the network will pervasively have to be operated, and planned, with time-varying thermal ratings (TVTRs) of transmission assets. Moreover, the smart grid will further facilitate the inclusion of demand response (DR) into the transmission network planning process. Therefore, this paper aims to discuss the need for, and implications of, adopting novel electricity network reliability planning and evaluation methodical approaches which consider flexible solutions (i.e., DR and TVTR). Subsequently, in order to encourage the adoption of these proposed approaches, the quantification of the benefits from increased, efficient and economical transmission utilization considering flexible concepts such as non-ageing TVTR, controlled-ageing of transmissions assets due to advanced operation of TVTR, and DR within transmission system are performed - and evaluated. The IEEE 24-bus RTS is used under sequential Monte Carlo simulation (SMCS) modelling of the reliability evaluation process in order to validate the proposed methodological enhancements and to evaluate network performance.

Keywords—adequacy, ageing, TVTR, thermal rating, demand response, reliability, network security, sequential modelling

\section{INTRODUCTION}

Network operators usually implement deterministic N-1 (or N-2 and/or N-D) outage criteria [1]. Through the implementation of any of these criteria, operators inherently rely on the pre-supposition that the network is not exposed to the risk of load curtailment following one, two or double circuit component failures. Nonetheless, this assumption is flawed because in reality the system is always exposed to risk of failure, and subsequently to customer outages in spite of the operator's ability to minimize this risk by implementing post fault corrective actions. Therefore, probabilistic methods have been proposed to tackle these problems [2]-[6].

In principle, the reliability criterion under probabilistic evaluation is superior to the deterministic one. Therefore, there is an imperative need to adopt probabilistic metrics to optimize and enhance network operation and planning [7]. The metrics of utilizing a probabilistic framework are rendered through their inherent ability to account for the vast range of uncertainties that surround the behavior of generation and transmission units. Thus historically, by simulating the system random behavior through techniques such as the Monte Carlo Simulation (MCS), reliability engineers have been able to accurately measure system's performance through the estimation of probabilistic indices of interest [8]. Consequently, much attention is now being placed on performing probabilistic reliability assessment by considering an advanced power system operating paradigm. This paradigm is associated with the proliferation of power systems technologies that promise to enhance its flexibility amid uncertain operating environments, for example, through the influx of renewable generation. Examples of flexible solutions include: ageing of overhead line (OHL) [9], demand side management [10], special protection systems with ICTs [11], wind farms [12], FACTs [12] and time varying thermal rating (TVTR) [13].

In this context, this work focusses on demonstrating the benefits of employing controlled ageing of OHLs, DR, and TVTR as cost effective alternative solutions worthy for consideration, and hence, modelling within transmission system planning and operational studies. For the implementation of these approaches, a more detailed modelling of OHLs properties is used; which contributes, to power system planning knowledge, a more precise calculation of network performance. This, resultantly, leads to a more accurate determination of the advantages of considering alternative flexible solution candidates for implementation on the transmission system. This, thus, provides the network operator with greater flexibility and reduced operational costs in the emerging smart grid paradigm.

\section{RELIABILITY EVALUATION AND NETWORK MODELLING CONSIDERING FLEXIBLE OPERATION REGIMES}

This section discusses how flexible operating regimes are modelled into the network for the performance of probabilistic reliability evaluation. These flexible regimes are simply an augmentation to the conventional standard probabilistic reliability evaluation process which is well understood by the modern power system planner and/or operator [8].

\section{A. Conventional Reliability Evaluation}

The traditional techniques employed by system planners and operators toward reliability evaluation fall into one of two categories: sequential or non-sequential [1]. Simply stated, the sequential reliability evaluation technique enables planners and operators to simulate stochastic power system behavior chronologically; the non-sequential does not. Consequently, the sequential technique produces results with relatively higher accuracy. Conversely, although the non-sequential technique is less accurate, it is computationally faster. Therefore, selecting which of the two techniques to utilize will be based upon making a compromise; solution accuracy 
against computational speed. In this paper the sequential technique is adopted; this sampling technique samples the exponential probability density function $f(t)=\lambda e^{-\lambda t}$ of power system components. A summary rendering of this application is discussed as follows [1]:

Step 1: All components are assumed to be initially up

Step 2: Then the duration of each component staying up is sampled from its reliability distribution function. Therefore, the time-to-failure (TTF) is sampled via the formulation in where $U$ is a randomly generated number from a uniform distribution lying between $[0,1]$ and $\lambda$ is taken as the failure rate of the component.

$$
\mathrm{TTF}=-\frac{1}{\lambda} \ln (1-\mathrm{U})
$$

Step 4: Similarly if a component is down, it's time to repair (TTR) is sampled in the same way as in. However, in this context; and $\mu$ will replace $\lambda$ as the repair rate of the component. In this step power flow and optimal power flow computational analyses [1,2] are performed in order to compute a desired reliability index. The reliability index used in this paper to evaluate the reliability of implementing (as well as not implementing) DR and TVTR is the energy expected not supplied (EENS, MWh/y) and is given by (2).

$$
E E N S=\sum_{i=1}^{N L}\left(\sum_{s e F_{i}} P(s) C(s)\right) T_{i}
$$

$\mathrm{C}(\mathrm{s})$ is the load curtailment in state $\mathrm{s}$

$\mathrm{P}(\mathrm{s})$ is the probability of system state $\mathrm{s}$

$\mathrm{NL}$ is the number of load levels

$\mathrm{T}_{\mathrm{i}}$ is the total number of the one period cycle (hours)

$F_{i}$ is the set of all system states associated with load curtailment

Step 5: Steps 2 and 3 are repeated over the duration of the system's mission time to create an array of system states in a chronological fashion; and to more importantly generate a sufficient number of states worthy of estimating the true EENS, within acceptable tolerances. Ascertaining this mandate requires the calculation of the EENS variance, and the coefficient of variation (COV) as defined by (3).

$$
C O V=\frac{\sqrt{\operatorname{Var}(E(F(x)))}}{E(F(x))}
$$

$\operatorname{Var}(\mathrm{E}(\mathrm{F}))$ is the variance of the estimated index.

Thus, in this narrated method (steps 1 to 5), descriptions of the system state for the whole time span are obtained allowing for the desired adequacy or security index (in the case of this paper, EENS) to be calculated.

\section{B. TITR Modelling}

Many transmission companies usually use a fixed thermal rating for short-term and long-term planning studies, which is calculated assuming extreme weather conditions and maximum conductor temperature, which can be tolerated by the OHL system (to prevent conductor annealing and eclipsing the maximum permitted sag limit). The fixed thermal rating is usually calculated for summer, due to higher ambient temperatures, which considerably affects the maximum capacity of the line. In this paper TITR fixed thermal rating models is implemented based on $40{ }^{\circ} \mathrm{C}$ ambient temperature, $0.61 \mathrm{~m} / \mathrm{sec}$ wind speed perpendicular to the conductor and 60 ${ }^{\circ} \mathrm{C}$ and $75{ }^{\circ} \mathrm{C}$ conductor temperature for the normal and emergency operation respectively.

\section{TVTR Modelling}

The reliability evaluation method previously defined (in section A) does not account for the time-varying nature of transmission system assets, but instead models them in a timeinvariant fashion-which conceals the true transmission capacity. Therefore, in order to determine the true transmission capacity that is available on a given network, the methodology to incorporate time-varying thermal ratings (TVTRs) used in this work is taken from [13], which uses an iterative algorithm that considers the TVTR in an annual basis. This behavior is augmented to the process of step 4 of the discussed reliability evaluation methodology in section A

More explicitly, the annual chronological load curve can be used with the annualized TVTR values. In this study, to foster computational efficiency, only the lines that are critically loaded are modelled with TVTR capability. This is realized by considering only steady-state conductor temperature calculations for the OHL adequacy. This is a justifiable consideration because the smallest unit of time being simulated in this study is an hour; which is in keeping with the ubiquitous common practice by power system reliability planners [8].

Furthermore, in order to identify the critically loaded lines, two case studies are implemented: one assuming the system is intact and the other assuming unexpected events occur, e.g. line outages. It should be stressed that in the analysis all the OHLs of the study network have been assumed (for computational burden and analytical relief, and lack of area segmented data) to be exposed to the same ambient conditions which influence the TVTRs.

In detail, the TVTR model proposed in [13] is used to represent the real transmission capacity that is available on a given network. The wind speed ( Vm), direction (Kangle), and ambient temperature ( $\mathrm{Ta}$ ) are used for the modeling of the OHL adequacy. The hourly weather data of 5 years from 1997 to 2001 were obtained from BADC Met office MIDAS stations for Aonach UK area [14]. In order to simplify the simulations the analysis was performed only for a year using the average value of each year for the hourly measurements $\left(\mathrm{V}_{\mathrm{m}}, \mathrm{K}_{\mathrm{a}}, \mathrm{T}_{\mathrm{a}}\right)$. Therefore, a single year of average values is used for the sequential analysis.

\section{Ageing-based TVTR Modelling}

There exists a strong relationship between TVTR and a conductor's operating temperature [15], [16]. This is shown in 


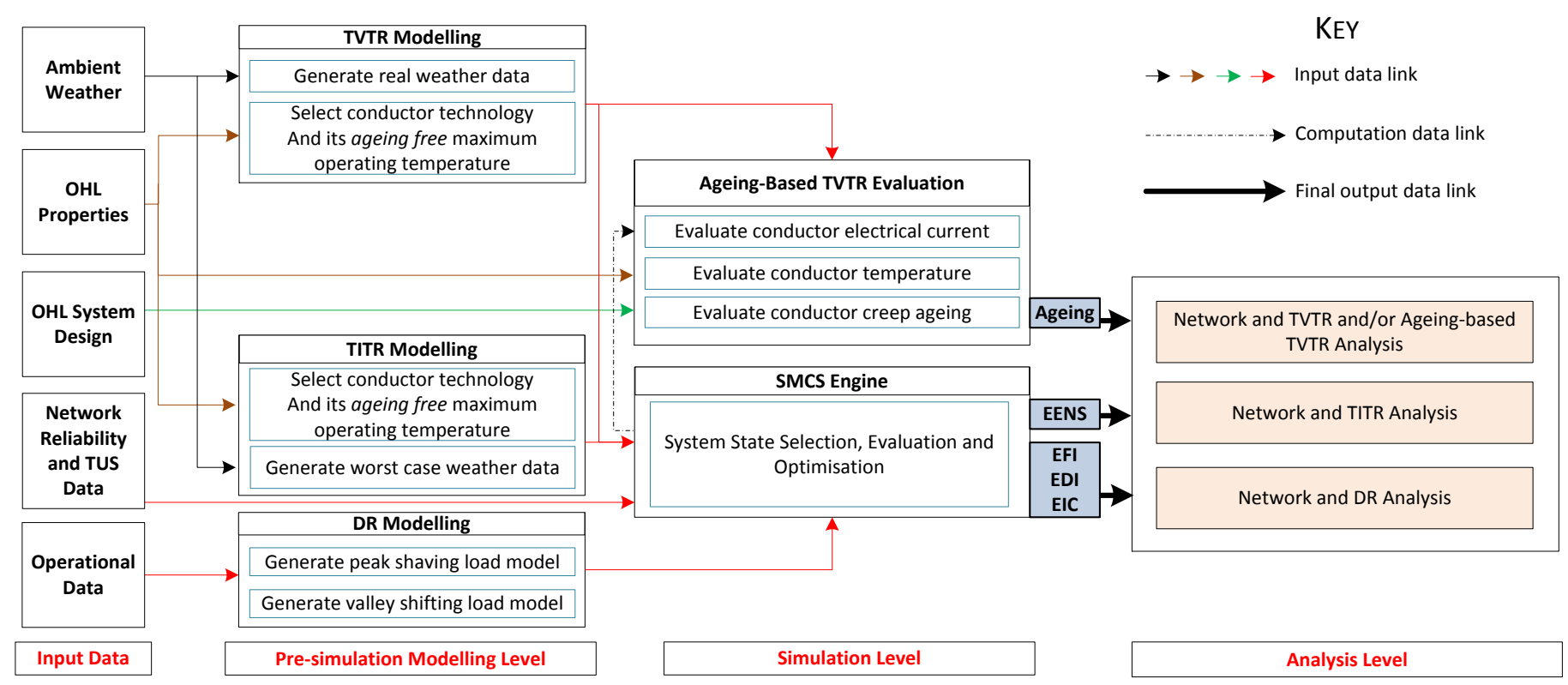

Fig. 1. Flow chart linking the TVTR, TITR and DR Modelling processes into the traditional SMCS reliability evaluation tool

(4). A complete description of (4) is found in [16]. It is sufficing to mention that, the speed of the wind, $V_{w}$, and its prevalence angle, $\theta$, of approach, the ambient temperature, $T_{a}$, surrounding it, the amount of solar radiation falling upon it, $Q_{s}$, and its resistance, $R\left(T_{c}\right)$, are important parameters to consider when evaluating TVTR.

$$
T V T R=\sqrt{\left[Q_{r}\left(V_{w}, T_{a}, T_{c}\right)+Q_{c}\left(T_{a}, T_{c}\right)-Q_{s}\right] / R\left(T_{c}\right)}
$$

When conductor temperature $\left(T_{c}\right)$ is below a particular threshold (e.g. $75^{\circ} \mathrm{C}$ or $95^{\circ} \mathrm{c}$ - depending upon the conductor technology type [3]), its material properties will not change. However, when a conductor operates above the aforesaid thresholds, then its material properties will change. This is termed as annealing [15] and accelerates the ageing process of the conductors. For this reason, operating conductors at temperatures elevated beyond the ageing thresholds has been omitted from transmission planning studies. However, ageing is a slow process; and if well controlled, transmission planners can suggest implementing higher TVTR ratings that can afford a certain amount of ageing. The methodology employed in this paper to model ageing is adopted from [17] and implemented into step 4 of the process narrated in Section A.

\section{E. DR Modeling}

The integration of DSM strategy on the transmission system operation and planning process has proven to be cost effective, and at the same time has shown to improve the security and reliability of the system. As a result, much attention is now being placed on performing probabilistic reliability assessment by considering DR programs in a composite power system operating paradigm. In this work, a two-step incentive based DR that considers peak shaving and valley shifting (PSVS) as well DR regimes are used (and augmented to step 4 of the process in section A). In addition, the original load curve is given by [18] and its sequential modelling is set (in hours) for three seasons from 8400 to 8568 hour (51 week) for winter analysis, from 3697 to 3864 for summer analysis and from 5377 to 5544 for spring analysis. The novelty of the DSM used is that it considers the frequency and the duration of the interruptions as well as the interruption cost of the customers at the load points. These factors are identified assuming, firstly, the occurrence of unexpected events, e.g. line, generator outages, and secondly the operation of the IEEE RTS without DSM strategy. In this study, all consumers are assumed to participate voluntarily in the DSM program; while no penalty is given if they do not comply with the DR rules (shift/ fill the load at off-peak times). Consumers' motivation relies on the fact that they receive prior notice either during peak demand or security violation. Consequently, the socioeconomic effect of probable loss of supply is substantially reduced. The implementation of the model is extensively discussed in [10].

The index used in this paper to evaluate the reliability of implementing DR at each load point is the expected frequency customer interruption index (EFI), expected duration customer interruption index and the expected interruption cost index given by (eq. 5-7).

$$
\begin{aligned}
& E F I_{j}=\frac{\sum_{m=1}^{N_{t}} N I_{m}}{\text { Samples }} \\
& E D I_{j}=\frac{\sum_{m=1}^{N_{t}} N I_{m} \times D i_{m}}{\text { Samples }^{\text {Di }}} \\
& \text { EIC }_{j}=\frac{\text { EENS }_{j} \times \text { VOLL }}{\text { Simulation year }}
\end{aligned}
$$


where,

NI:

Di:

$\mathrm{Nt}$ :

VOLL

Number of customer's interruptions

Duration of customer's interruption

Interruption duration, lasting up to 24 hours

Value of Loss of Load (\$/KWh)

\section{TEST SYSTEM}

The test system (Fig. 2) upon which the flexible transmission planning solutions (as holistically illustrated through the flow chart in Fig. 1) are investigated is the IEEERTS 24 bus system [4]. This system is characterized by detailed network reliability data; hence, in this regard, this system meets the requirements that allow for the methodologies earlier presented to be tested. This system contains 38 lines and 32 generators of varying types such as hydro, coal/steam, nuclear. The total peak load of the system is $2850 \mathrm{MW}$, while the total generating capacity is $3405 \mathrm{MW}$. The sequential MC algorithms (under the discussed flexible solution models) and the IEEE-RTS 24 bus test system were developed in Matlab using modified Matpower for the power flow calculations [19]. The MIPS solver is used for the ACOPF in Matpower with objective functions being the minimization of the load curtailment and the minimization of the generation costs.

\section{CASE STUDY DESCRIPTIONS}

\section{A. TVTR Analysis}

Probabilistic reliability evaluation is implemented by using sequential MC in order to incorporate the DR model. In particular, the method considers that transmission lines are represented by a two state model: up and down. Exponential distribution function is used to identify the mean values of time to failure and time to repair of the lines and generators.

Under normal operation (no outages occur) the conductor temperature, $\mathrm{T}_{\mathrm{c}}$, is set at $60^{\circ} \mathrm{C}$ based on avoidance of the conductor annealing [20]. A line is defined to be at emergency state, when another transmission line connected on the same bus (as the one in emergency state) has failed. In cases a failure of a line does not result in a credible outage then lines are considered as being at normal operation state. In the present study under emergency operation the maximum conductor operating temperature is considered to be $75^{\circ} \mathrm{C}$.

\section{B. Ageing-Based TVTR Analysis}

As earlier mentioned, operating conductors at temperatures elevated beyond the ageing thresholds has been omitted from transmission planning studies. However, ageing is a slow process; and if well controlled, transmission planners can suggest implementing higher TVTR ratings that can afford a certain amount of ageing. Therefore, these higher TVTR ratings can be termed as emergency ratings; schedulable only under special emergency situations. To showcase this benefit the system is modified to be characterized by two types of emergency TVTR ratings: (1) the long term emergency (LTE); and (2) the short term emergency (STE). The duration of STE is controlled to 15 minutes, whereas LTE is controlled to 24

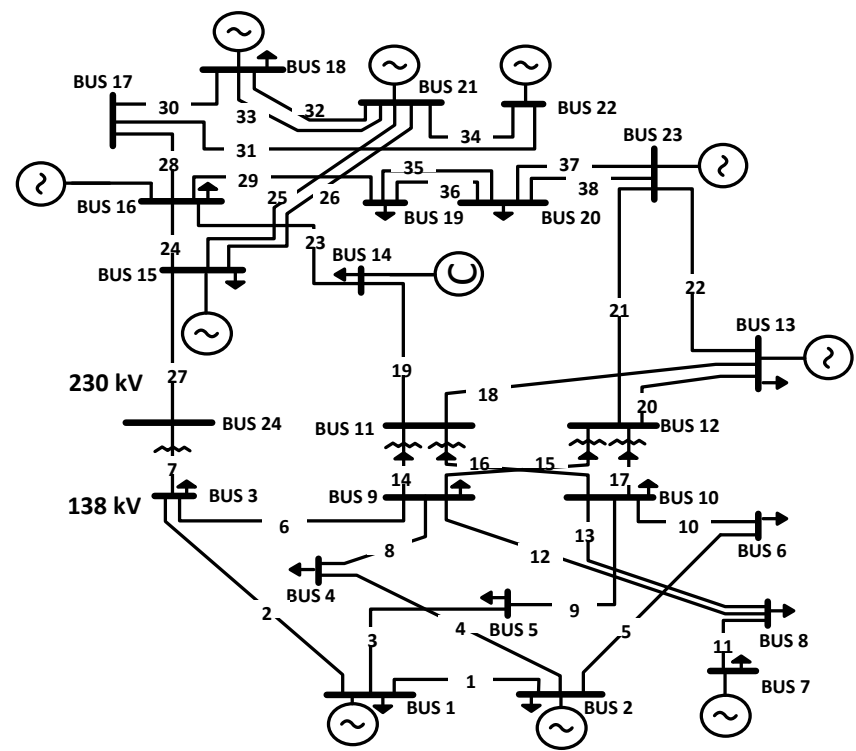

Fig. 2. Test system schematic

hours for the IEEE-RTS. However, to perceive how the selection of the appropriate emergency rating and duration might influence system reliability-adequacy, this paper engages a comprehensive study to ascertain the best STE and LTE ratings earlier narrated by varying the TVTR magnitudes between $[1,1.3] \mathrm{pu}$ multiples of the normal rating for the IEEE-RTS 24 bus network. Moreover, for these studies, the demand is set to $1.5 \mathrm{pu}$ of nominal; and generation capacity is set to $2 \mathrm{pu}$.

\section{DR Analysis}

The additional scenario with increased load to $1.2 \mathrm{pu}$ its normal load; with $0.55 \mathrm{pu}$ and $0.6 \mathrm{pu}$ (for the $138 \mathrm{KV}$ and $230 \mathrm{KV}$ respectively) is also used in order to determine the importance of each load point and set weight indexes with the view to facilitate network operators for practicing DSM programs (baseline). The load profile consists of the 51 winter peak weak, 23 summer peak week and 16 spring peak week. This interruption based study is performed for both intact and contingent network scenarios using DR under TITR operating regime.

\section{CASE STUdY RESUlTS}

\section{A. Ageing-Based TVTR Analysis}

In this section, results to the case study to investigate the effects of utilizing either STE or LTE; rates or magnitudes are discussed. Results are plotted in Fig. $3 \mathrm{i}$ and ii, and Fig. 4.

Fig.3.i shows the influence of only employing varied STE magnitude ratings for a total of 15 minutes. Consequently, it can be observed that the optimal rating is $1.25 \mathrm{pu}$. Moreover, it can be seen that an increase above this value (i.e., to $1.3 \mathrm{pu}$ ) increases the system's un-reliability level by $\sim 1.35 \%$. This is because the system experiences increased voltage stability problems when it attempts to load the critical lines beyond $1.25 \mathrm{pu}$ (i.e. at $1.3 \mathrm{pu}$ ) for 15 minutes. Conversely, utilizing magnitudes below $1.25 \mathrm{pu}$ amputates the system's supply adequacy during the 15 minutes schedule. Thus the adequacy 
TABLE I.
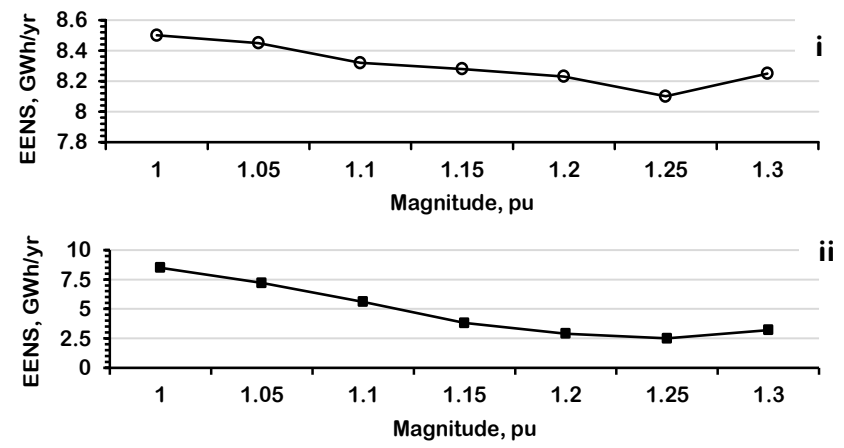

\begin{tabular}{|c|cccccc|}
\hline \hline \multirow{2}{*}{$\begin{array}{c}\text { Reliability } \\
\text { indices }\end{array}$} & \multicolumn{2}{|c}{ Winter } & \multicolumn{2}{c}{ Summer } & \multicolumn{2}{c|}{ Spring } \\
\cline { 2 - 7 } & without & DR & $\begin{array}{c}\text { without } \\
\text { DR }\end{array}$ & DR & $\begin{array}{c}\text { Without } \\
\text { DR }\end{array}$ & DR \\
\hline EENS(MW) & 851.24 & 601.36 & 180.048 & 104.602 & 101.95 & 52.1139 \\
ETOC(K\$) & 21299 & 12058 & 2778 & 593.7 & 61530 & 45777 \\
EDI & 0.1268 & 0.121 & 0.0495 & 0.0288 & 0.0283 & 0.0188 \\
EFI & 0.033 & 0.023 & 0.0181 & 0.0098 & 0.0079 & 0.0067 \\
\hline \hline
\end{tabular}

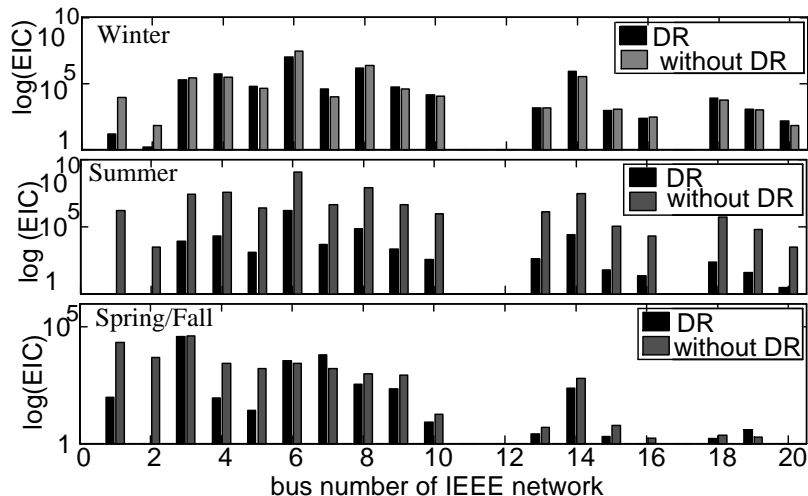

Fig. 5. Comparison of Expected Interrupted Cost (EIC)

improvement between operating at $1 \mathrm{pu}$ and $1.25 \mathrm{pu}$ is $3.57 \%$. The EENS at 1.25 is $8.1 \mathrm{GWh}$ and $1 \mathrm{pu}$ is $\sim 8.5 \mathrm{GWh}$.

Fig 3.ii shows the influence of solely employing the LTE rate by varying its magnitude whilst keeping its duration constant at 24 hours. Firstly it must be observed that the EENS at $1.25 \mathrm{pu}$ is recorded to be $2.9 \mathrm{GWh}$. In comparison with the STE case, this demonstrates a vast improvement in the region of $\sim 64.2 \%$. Clearly, this huge improvement in reliability cites the huge importance of duration on reliability for this system's stipulated conditions. This is simply because as the emergency rate is increased, the system is able to serve demand for much longer durations.

Furthermore assessing the plot in Fig.3 ii with itself, one can clearly observe the impact of LTE magnitude; this is because between 1 pu and 1.25 pu a $65.8 \%$ improvement is gained. Further observance shows that similar to the STE case (Fig. 3 i) when the system is loaded beyond 1.25 pu the unreliability level increases with respect to the level at $1.25 \mathrm{pu}$. This degradation value is $\sim 9.37 \%$ at $\sim 3.1 \mathrm{GWh}$ when operated at $1.3 \mathrm{pu}$. These studies thus far have demonstrated the efficacy of increasing the TVTR emergency rating to 1.25 $\mathrm{pu}$, for a duration of 24 hours. The corresponding system age (at $1.25 \mathrm{pu}$ ) has also been shown in Fig 4 to be about 182 Hours/year. This value can be used to make asset management decisions which will aid to approve operating the transmission assets at a higher TVTR. Thus in summary, the studies present an improved and quantitative approach to aid the selection, and adoption of, higher TVTRs (when compared with the traditional conservative approach based on engineering judgments solely) through an asset management approach.

\section{A. DR-Based TITR Analysis}

The results of the case studies indicate network improvement following DR operation scheme. Table I illustrates the reliability performance of the network considering three case studies. Each case study includes network performance indices for each season of the year.

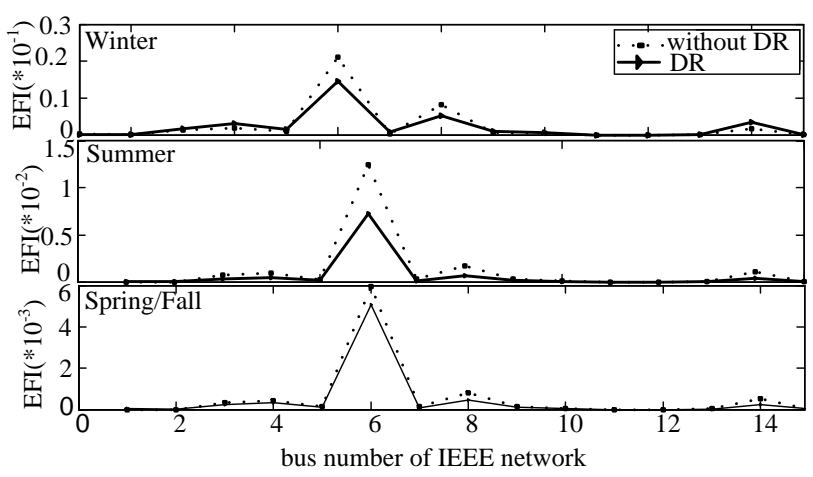

Fig. 6. Comparison of EFI for the different seasons

The analysis of each season is conducted for both the baseline and DR as a corrective action for post fault analysis with TITR operation regime. It is evident from Table I that DR renders the most secure and economic scenario. In particular, the TITR and DR implementation in winter resulted in $29.3 \%$ lower EENS than the baseline (without DR model). The corresponding EENS improvement for summer is $41.9 \%$ while in spring/fall EENS is $48.8 \%$ less than the baseline.

This is mainly due to the ability of DR to alleviate the overloading of OHLs. The expected total Operating Cost (ETOC) of the network, which considers both generation cost and cost of interruptions, decreases significantly, when DR is implemented as during contingent conditions both cheaper generators and demand reduction can be utilized. Table I indicates the actual values of TOC, EENS, EDI and EFI. In particular, the TOC is decreased by $43 \%, 78 \%, 25 \%$ under DR for winter, summer, spring/fall respectively.

This is a result of the more economic DR practice, which allows the expensive generators to produce less or even zero power during peak time. In addition, when the EDI is 
considered, it can be inferred from the table that although EDI during the summer decreases significantly under DR regime (i.e., from 0.0495 to 0.0288 ), in the winter, however, the EDI difference is small-in the range of 0.005 . This indicates that the DR mainly affects the magnitude of the loss of load at a load point and not the duration of the interruption during winter, whereas in summer it analogically improves both indices.

Fig.5 compares the Expected Interruption Cost (EIC) at each load point under TITR with (DR) and without DR (baseline) for each season of the year. Considering winter scenario, EIC is higher in the baseline except for B7, B20 due to no utilization of DR and fixed thermal rating of the OHL that are connected to this bus. In detail, EIC of B1, B2, B6 have been substantially reduced due to the DR model, while a slight difference is seen in EIC of B3, B5, B9, and B13. On the contrary,

EIC is notably higher in the baseline through all the load points in summer analysis. In some cases such as in B1, B2 and B20, DR utterly eliminated customers' interruption, which is mainly because of the small amount of EENS occurring at these buses (27MW, $0.1 \mathrm{MW}, 0.2 \mathrm{MW}$ respectively). Correspondingly, interruption costs are entirely alleviated in B2, B15, B18 under spring/fall scenario, which is a significant DR effect especially for $\mathrm{B} 2$ with $\mathrm{K} \$ 2,74$ EIC under the baseline. However, it is indicated that EIC is higher in B6 under DR which is due to security violations DR causes to the network in the event of generation and transmission lines outages.

The EFI index is illustrated in Fig.6 at each load point under TITR with (DR) and without DR (baseline) for each season of the year. The results of the case study indicate that B6 is the most frequently interrupted bus, due to lack of generation unit connected to this bus, as well as the high failure rates of L5 and L10 lines at which the bus is connected to. In summer for instance, the difference is higher than winter and more particularly by $41.6 \%$ comparing the proposed DR with the baseline.

In summary, EFI follows the same pattern for both summer and winter studies with the stark contrast of winter having double frequency of interruptions compared to summer. Similarly, spring/fall study show decrease of EFI (by almost 3.5 times less than winter) under both DR and the baseline operation regime. Overall, DR largely affects the frequency of the interruptions in the most critical load points of the network under the different seasons, whereas in some buses the effect is negligible due to minor EFI they demonstrate.

\section{CONCLUSION}

This paper presents an analysis of network performance when both DR and ageing regimes in conjunction with TVTR are available to the operator. The results show that DR and TVTR increase network reliability and reduce operation costs providing benefits to both customers and network operators. When DR with TITR operation is implemented, the total EIC of the 24-bus network is reduced by $59 \%, 74 \%$ and $37.8 \%$ for winter, summer and spring respectively. It is highlighted that in summer the EENS of the network is reduced by $41.9 \%$ when DR with TITR are considered. This signifies the importance of the DR operating regime in the transmission system.
Additionally, it has been quantitatively shown that increasing TVTRs to allow for controlled ageing renders the potential to increase power system reliability by up to $65.8 \%$. Indeed these cited benefits are colossal and strongly encourage power system planners to adopt these presented methodologies in order to flexibly plan power systems with minimal investment - in comparison to the building of new lines, which in additional to their high capital investment cost are increasingly witnessing public objection.

\section{REFERENCES}

[1] L. Söder, J. Douglas, and A. Ekwue, "Study on the Technical Security Rules of the European Electricity Network," Stockholm, 2006

[2] W. Li and P. Choudhury, "Probabilistic Transmission Planning," IEEE Power Energy Mag., vol. 5, pp. 46 - 53, 2007.

[3] J. D. McCalley et al., "Probabilistic security assessment for power system operations," in Power Engineering Society General Meeting, 2004 , pp. $212-220$.

[4] J. M. Arroyo and N. Alguacil, "A Risk-Based Approach for Transmission Network,” IEEE Trans. Power Syst., vol. 25, no. 3, pp. 1759-1766, 1995.

[5] D. S. Kirschen, K. R. W. Bell, D. P. Nedic, D. Jayaweera, and R. N. Allan, "Computing the value of security," in IEE ProceedingsGeneration, Transmission and Distribution, 2003, vol. 150.

[6] A. Kapetanaki and K. Kopsidas, "MOPSO using Probabilistic and Deterministic criteria based on OHL's Thermal Ratings," in PSCC, 2014.

[7] R. Moreno, D. Pudjianto, and G. Strbac, "Transmission Network Investment with Probabilistic Security and Corrective Control," IEEE Trans. POWER Syst., vol. 28, no. 4, pp. 3935-3944, 2013.

[8] R. Billinton and W. Li, Reliability Assessment of Electrical Power Systems Using Monte Carlo Methods. N.Y: London:Plenum, 1994.

[9] C. Tumelo-Chakonta and K. Kopsidas, "Assessing the Value of Employing Dynamic Thermal Rating on System-wide

Performance," in ISGT Europe, 2011, pp. 1-8.

[10] A. Kapetanaki and K. Kopsidas, "Reliability Evaluation of DR and TVTR considering the Cost of Interruptions," in ISGT 2014.

[11] B. Falahati, Y. Fu, and L. Wu, "Reliability Assessment of Smart Grid Considering Direct Cyber-Power Interdependencies," IEEE Trans. POWER Syst., vol. 3, no. 3, pp. 1515-1524, 2012.

[12] R. Billinton and W. Wangdee, "Reliability-Based Transmission Reinforcement Planning Associated With Large-Scale Wind Farms," IEEE Trans. Power Syst., vol. 22, no. 1, pp. 34-41, Feb. 2007.

[13] A. Kapetanaki, K. Kopsidas, C. Tumelo-Chakonta, and M. Buhari, "Network Planning Evaluation Implementing Time Varying Thermal Ratings," in PMAPS, 2014.

[14] "http://badc.nerc.ac.uk/data/ukmo-midas/WPS.html."

[15] "IEEE Guide for Determining the Effects of High-Temperature Operation on Conductors, Connectors, and Accessories," IEEE Std 1283-2004, pp. 1-28, 2005.

[16] "IEEE Standard for Calculating the Current-Temperature of Bare Overhead Conductors," IEEE Std 738-2006 (Revision Std 7381993), pp. c1-59, 2007.

[17] C. Tumelo-Chakonta and K. Kopsidas, "Assessing Optimal Conductor Utilization under N-1 Overload Security Studies," pp. 16.

[18] C. Fong, S. Haddad, and D. Patton, "The IEEE Reliability test system-1996," vol. 14, no. 3, 1999.

[19] R. D. Zimmerman, E. M.-S. Carlos, and D. Gan, "MATPOWER:A MATLAB Power System Simulation Package, Version 3.1b2, User's Manual." Power Systems Engineering Research center(PSERC), Tech. Rep. 2006, New York, 2011.

[20] K. Kopsidas, S. M. Rowland, and B. Boumecid, "A Holistic Method for Conductor Ampacity and Sag Computation on an OHL Structure," IEEE Trans. Power Deliv., vol. 27, no. 3, 2012. 